Original Article

\title{
Intrarater reliability of measuring the patella position by ultrasonography in weight-bearing condition
}

\author{
Chia Lin $\mathrm{Chen}^{1,2)}$, Chu Ling Lo ${ }^{3)}$ *, Kai Chu Huang ${ }^{1)}$, Chen Fu Huang ${ }^{2)}$ \\ 1) Department of Physical Therapy, Tzu Hui Institute of Technology, Taiwan \\ 2) Department of Physical Education, National Taiwan Normal University, Taiwan \\ 3) Department of Physical Medicine and Rehabilitation, Cheng Ching Hospital Chung Kang Branch: \\ No. 966, Sec. 4, Taiwan Blvd., Xitun District, Taichung City 407, Taiwan
}

\begin{abstract}
Purpose] The aim of this study was to determine the intrarater reliability of using ultrasonography as a measurement tool to assess the patella position in a weight-bearing condition. [Subjects and Methods] Ten healthy adults participated in this study. Ultrasonography was used to assess the patella position during step down with the loading knee in flexion $\left(0^{\circ}\right.$ and $\left.20^{\circ}\right)$. The distance between the patella and lateral condyle was measured to represent the patella position on the condylar groove. Two measurements were obtained on the first day and the day after 1 week by the same investigator. [Results] Excellent intrarater reliability, ranging from 0.83 to 0.93 , was shown in both conditions. Standard errors of the measurements were $0.5 \mathrm{~mm}$ in the straight knee and $0.7 \mathrm{~mm}$ in the knee flexion at $20^{\circ}$. Minimal differences in knee flexion at $0^{\circ}$ and knee flexion at $20^{\circ}$ were $1.5 \mathrm{~mm}$ and $1.9 \mathrm{~mm}$, respectively. [Conclusion] Ultrasonography is a reliable assessment tool for evaluating the positional changes of the patella in weight-bearing activities, and it can be easily used by practitioners in the clinical setting.

Key words: Patella position, Reliability, Ultrasonography
\end{abstract}

(This article was submitted Jun. 1, 2017, and was accepted Jul. 22, 2017)

\section{INTRODUCTION}

Patella malalignment or maltracking is a risk factor in musculoskeletal disorders, such as anterior knee pain, patellofemoral pain syndrome, and iliotibial band syndrome ${ }^{1-5}$. Alignment represents the static position of the patella on the trochlea, whereas tracking means the dynamic motion of the patella on the femur. Imbalanced forces on the patella, which are generated by the surrounding soft tissue, such as vastus medialis oblique weakness and iliotibial band or lateral retinaculum tightness, will cause malalignment of patella. Changes in lower limb alignment also cause patella maltracking, such as femoral or tibial rotation and excessive subtalar joint pronation ${ }^{1,5-9}$. Patella malalignment and maltracking may increase contact pressures on the lateral facet of the patella ${ }^{7}$, which damages cartilage and surrounding soft tissues, and causes pain, apprehension, and giving way ${ }^{1)}$.

To accurately evaluate the patella position relative to the femoral groove is very important for physical therapists in clinical practice when choosing preventive or treatment strategies. Various methods have been proposed to assess the patella orientation in previous studies ${ }^{10-14}$. However, which method has the best reliability and can be easily to apply in the clinical setting is still controversial. McConnell was the first to used palpation and visual estimation to assess the patella position ${ }^{3,5)}$, which showed poor to fair intrarater and interrater reliabilities ${ }^{10)}$. Additionally, its validity has been questioned ${ }^{11)}$. Some researchers have marked the epicondyles and mid-patella position and then used tape or calipers to assess the medial and lateral distances to represent the patella position ${ }^{12-14)}$. Results of intrarater reliability of those studies varied because of

*Corresponding author. Chu Ling Lo (E-mail: lochuling@hotmail.com)

(C2017 The Society of Physical Therapy Science. Published by IPEC Inc.

(c) $($ ) $($ This is an open-access article distributed under the terms of the Creative Commons Attribution Non-Commercial No Deriva-

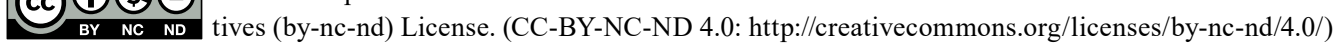


experience of the examiner to the test and showed poor agreement with magnetic resonance imaging (MRI) findings. Recent studies that used ultrasonography as an assessment tool showed good results ${ }^{15}{ }^{16}$. However, most of these studies obtained the measurements in non-weight-bearing conditions and did not control for confounding factors, such as lower limb rotation, quadriceps contraction, or knee flexion ${ }^{1,17}$.

Alignment measures of the patella may be very different in an unloaded static position, such as in supine position and loaded activities that may exacerbate symptoms ${ }^{1)}$. It is important to assess the position of the patella in a weight-bearing condition that could provide accurate information for evaluating the treatment effect. Only a few studies have assessed the patella position in a weight-bearing condition; however, the methods were not convenient or easy to apply in the clinical setting. Shih et al. ${ }^{17)}$ used a functional knee brace with a linkage system mounted to the transepicondylar axis to measure the patella position in loading activities. Nevertheless, the authors did not take the tibia and foot alignment and the modified brace, which may affect movement control of the lower limbs, into consideration. Tennant el al. ${ }^{18)}$ used dynamic MRI to assess the patello-femoral tracking in a weight-bearing position. However, MRI is an expensive evaluation tool and unsuitable to repeatedly use in a clinic. The purpose of this study was to determine the intrarater reliability of measuring the patella position in a weight-bearing condition with knee flexion at $0^{\circ}$ or $20^{\circ}$ by ultrasonography and controlling for confounding factors.

\section{SUBJECTS AND METHODS}

Ten asymptomatic adults ( 6 females, 4 males) were enrolled in this study. Healthy participants with normal foot type aged between 20 and 65 years were included. A normal foot type was defined as a foot posture index $\geq 1$ and $\leq 6^{19)}$ that was evaluated by one physical therapist. Participants with a history of low back or lower limb pathology, neuromuscular diseases within 6 months, or a known pregnancy were excluded. Informed consent, which was reviewed by the Institutional Review Board of Cheng Ching Hospital, Taichung, Taiwan (HP170011), was signed by all participants.

Participants were instructed to stand on their dominant leg with the other leg rest on a $15-\mathrm{cm}$ step. To control lower limb rotation, participants should keep origin position in each trial. The dominant leg was determined by a ball-kicking test. Two different knee flexion angles (i.e., $0^{\circ}$ and $20^{\circ}$ ) of the dominant leg were measured. Participants were asked to stand as naturally as possible, and they were reminded not to hyper-extend the knee during the trial of $0^{\circ}$ knee flexion. To ensure the consistency of each trial, a weight meter was placed on the step and the number on weight meter should keep less than $5 \mathrm{~kg}$ during testing to control the loading proportion, which means participants should nearly fully load on the testing limb. Participants were instructed to keep their trunk upright, place their arms on both sides of their body, and look at the sign on the wall (Fig. 1(A)). They were allowed to practice the task so they felt comfortable with it. A goniometer fixed at $0^{\circ}$ or $20^{\circ}$ was attached on the medial side of the knee joint to ensure that the knee maintained the same angle during the measurements. The order of the trials was randomly assigned.

B-mode real-time ultrasonography (ALOKA ProSound 2, Hitachi Aloka Medical, Ltd., Mitaka-Shi, Japan) was used to measure the position of the patella. The 5-MHz linear array probe was placed on the lateral edge of the superior border of the patella with water-soluble transmission gel in between the probe and the skin. The distance between the lateral point of the patella and medial border of the lateral condyle was measured by the on-screen caliper as the patella-condyle distance $(\mathrm{PCD})^{17)}$ (Fig. 1(B)). The position of the probe was marked on the skin to ensure measurement consistency. Two trials of measurements were done on the first day and the day after 1 week, and three repetitive measures of each condition were recorded in each trial by the same physical therapist.

SPSS version 20 software (IBM Corp., Armonk, NY, USA) was used to perform statistical analyses. Descriptive data are reported as means and standard deviations. The intraclass correlation coefficient (ICC) with a 95\% confidence interval represents the reliability of the PCD measurement of the two conditions. Standard errors of the measurements (SEM) and minimal difference (MD) values were calculated by using the following formulas: $S E M=S D * \sqrt{1-I C C}$ and $M D=1.96 * \sqrt{2} * \mathrm{SEM}$. The SEM is an index that can be used to define the difference needed between separate measures of a subject for the difference in the measures to be considered real. MD values can be used to determine threshold values for a real change ${ }^{20)}$.

\section{RESULTS}

Ten participants were recruited. No discomfort or pain was reported by any participant during or after the test. Patients' demographic characteristics are presented in Table 1.

Intrarater reliability, SEM, and MDs of the two conditions are summarized in Table 2. ICC values in both testing angles are excellent. Besides, SEM and MD showed similar results.

\section{DISCUSSION}

In our study, we observed excellent intrarater reliability of measuring the static patella position in weight-bearing conditions (straight leg or knee flexion at $20^{\circ}$ ) by using ultrasonography. ICC values were 0.93 and 0.83 in of knee flexion at $0^{\circ}$ and $20^{\circ}$, respectively. Results of our study were similar to those of previous studies that also measured the patella position by using ultrasonography in both non-weight bearing and weight-bearing conditions. Herrington et al. ${ }^{15,21)}$ reported excellent 

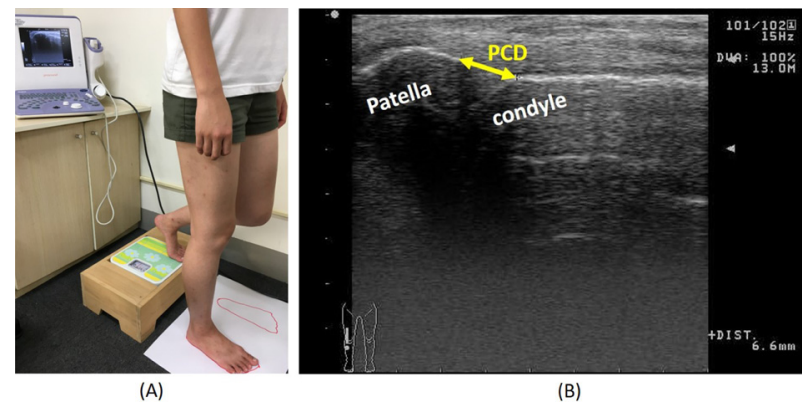

Table 1. Demographic characteristics of participants

\begin{tabular}{lc}
\hline & Results (mean $\pm \mathrm{SD}$ ) \\
\hline Gender, male/female & $4 / 6$ \\
Age (years) & $22.7 \pm 2.2$ \\
Height $(\mathrm{cm})$ & $168.9 \pm 8.7$ \\
Weight $(\mathrm{kg})$ & $59.5 \pm 11.3$ \\
\hline
\end{tabular}

SD: standard deviation

Fig. 1. (A) Test position for measuring patella-condyle distance. (B) Ultrasonography image of patella-condyle distance PCD: patella-condyle distance

Table 2. The ICC, SEMs, and MD of the amount of the patella-condyle distance (PCD)

\begin{tabular}{lccc}
\hline & ICC $(95 \% \mathrm{CI})$ & SEMs $(\mathrm{mm})$ & MD $(\mathrm{mm})$ \\
\hline PCD-Knee flexion $0^{\circ}$ & $0.93(0.75-0.98)^{*}$ & 0.5 & 1.5 \\
PCD-Knee flexion $20^{\circ}$ & $0.86(0.55-0.96)^{*}$ & 0.7 & 1.9 \\
\hline
\end{tabular}

*Statistical significance $(\mathrm{p}<0.05)$

reliability with an ICC value ranging from 0.87 to 0.99 in a non-weight bearing task. However, another comparable study by Shih et al. ${ }^{17)}$ showed poor to excellent intrarater reliability that ranged from 0.29 to 0.9 in weight-bearing activities; this result may be related to different measurement methods and the angle of knee flexion.

SEMs were $0.5 \mathrm{~mm}$ in the straight knee and $0.7 \mathrm{~mm}$ in the knee flexion at $20^{\circ}$, and the minimal differences in knee flexion at $0^{\circ}$ and knee flexion at $20^{\circ}$ were $1.5 \mathrm{~mm}$ and $1.9 \mathrm{~mm}$, respectively, in the present study. Herrington and Pearson ${ }^{21)}$ reported a slightly greater SEM of $3.6 \mathrm{~mm}$ and similar minimal difference of $1.3 \mathrm{~mm}$ in a non-weight bearing condition. Herrington and Pearson used electrical stimulation to induce a tetanic muscle contraction. However, submaximal active muscle contraction was used in our study. Different level of quadriceps contraction in weight-bearing or non-weight-bearing activities may cause the difference in the SEM.

According to previous studies, it had been proved that $25 \%$ of weight bearing is enough to engage the patella onto the condylar groove, and it did not cause an artifact to appear on the ultrasonogram while obtaining the measurement. In our study, we asked the participants to fully bear weight on their dominant leg during each trial. Increasing weight bearing would not change the alignment or contact area of the patellofemoral joint ${ }^{22,23)}$, as the contact area of the patellofemoral joint only changed with the angle of knee flexion ${ }^{22}$.

A straight knee condition and knee flexion at $20^{\circ}$ were chosen in this study. Tennant et al. ${ }^{18)}$ showed that the patella was slightly laterally displaced in knee hyperextension and then it returned to a central location during the initial $30^{\circ}$ of knee flexion. Patients with patellofemoral pain syndrome often complain of discomfort in the initial range of knee flexion while ascending or descending stairs ${ }^{3)}$. According to the results of a recent study, significant muscle imbalance was noted in knee flexion at $15^{\circ}$ and $60^{\circ}$ in a weight-bearing condition in participants with patellofemoral pain syndrome ${ }^{6}$. Elias and White ${ }^{1)}$ suggested that imaging studies of patellofemoral tracking should focus on the initial range of knee flexion. With the knee flexed beyond $45^{\circ}$, the patella fully engaged into the condyle groove of the femur. In our study, the PCD was measured in straight knee and knee flexion at $20^{\circ}$ to reveal the patella position changes in initial knee flexion.

No extra device was used in this study to measure the patella position in weight-bearing activities to prevent movement of the dominant lower limb from interfering, because the ultrasound transducer can stay perpendicular to the lateral surface of the patella. Shih et al. used an extra device to fix transducer which resulted in measurement error in the angle of the ultrasound transducer to the patella and poor $\mathrm{ICC}^{17}$. Compared to the image of the patella position in a non-weight-bearing condition, an image in a weight-bearing condition can reveal more realistic alignment change.

There were several limitations in this study. First, only static alignment of the patella was assessed, so dynamic tracking changes of the patella cannot be determined by the method we used. Second, only intrarater reliability was assessed in this study. Third, only asymptomatic participants were recruited in our study. Fourth, no direct measurement in quadriceps contraction in our study, so we cannot ensure the level of quadriceps contraction in each trial. In future study, electromyography (EMG) should be used to monitor the level of quadriceps contraction to ensure the consistency of each trail, and dynamic tracking changes of participants with patellofemoral pain should be investigated. The interrater reliability and validity of the assessment technique we used should be investigated in future. 
In conclusion, this study showed an excellent intrarater reliability and small SEM and minimal difference, which means the method we used was a reliable and sensitive measurement for detecting changes in the patella position in a weight-bearing task. In addition, measuring the patella position with ultrasonography can be easily applied in the clinic setting.

Compared to other imaging assessments such as MRI or computed tomography, ultrasonography is a non-radiation, non-invasive, cheaper, and reliable measurement tool, and it can be repeatedly applied to assess the patella position in weight-bearing activities. Accurately measuring the patella position in weight-bearing activities can provide more realistic information for practitioners when making a differential diagnosis and clinical decision.

\section{REFERENCES}

1) Elias DA, White LM: Imaging of patellofemoral disorders. Clin Radiol, 2004, 59: 543-557. [Medline] [CrossRef]

2) Kwon O, Yun M, Lee W: Correlation between intrinsic patellofemoral pain syndrome in young adults and lower extremity biomechanics. J Phys Ther Sci, 2014, 26: 961-964. [Medline] [CrossRef]

3) McConnell J: The management of chondromalacia patellae: a long term solution. Aust J Physiother, 1986, 32: 215-223. [Medline] [CrossRef]

4) Witvrouw E, Lysens R, Bellemans J, et al.: Intrinsic risk factors for the development of anterior knee pain in an athletic population. A two-year prospective study. Am J Sports Med, 2000, 28: 480-489. [Medline] [CrossRef]

5) McConnell J: Management of patellofemoral problems. Man Ther, 1996, 1: 60-66. [Medline] [CrossRef]

6) Kang JI, Park JS, Choi H, et al.: A study on muscle activity and ratio of the knee extensor depending on the types of squat exercise. J Phys Ther Sci, 2017, 29: 43-47. [Medline] [CrossRef]

7) Li G, Papannagari R, Nha KW, et al.: The coupled motion of the femur and patella during in vivo weightbearing knee flexion. J Biomech Eng, 2007, 129: 937-943. [Medline] [CrossRef]

8) Park JH, Kang SY, Choung SD, et al.: Effects of tibial rotation on Ober's test and patellar tracking. Knee, 2016, 23: 600-603. [Medline] [CrossRef]

9) Tiberio D: The effect of excessive subtalar joint pronation on patellofemoral mechanics: a theoretical model. J Orthop Sports Phys Ther, 1987, 9: 160-165. [Medline] [CrossRef]

10) Watson CJ, Propps M, Galt W, et al.: Reliability of McConnell's classification of patellar orientation in symptomatic and asymptomatic subjects. J Orthop Sports Phys Ther, 1999, 29: 378-385, discussion 386-393. [Medline] [CrossRef]

11) Powers CM, Mortenson S, Nishimoto D, et al.: Criterion-related validity of a clinical measurement to determine the medial/lateral component of patellar orientation. J Orthop Sports Phys Ther, 1999, 29: 372-377. [Medline] [CrossRef]

12) Tomsich DA, Nitz AJ, Threlkeld AJ, et al.: Patellofemoral alignment: reliability. J Orthop Sports Phys Ther, 1996, 23: 200-208. [Medline] [CrossRef]

13) McEwan I, Herrington L, Thom J: The validity of clinical measures of patella position. Man Ther, 2007, 12: 226-230. [Medline] [CrossRef]

14) Sacco IC, Onodera AN, Butugan MK, et al.: Inter- and intra-tester reliability of clinical measurement to determine medio-lateral patellar position using a pachymeter or visual assessment. Knee, 2010, 17: 92-95. [Medline] [CrossRef]

15) Herrington L, McEwan I, Thom J: Quantification of patella position by ultrasound scanning and its criterion validity. Ultrasound Med Biol, 2006, 32: 18331836. [Medline] [CrossRef]

16) Smith TO, Davies L, Donell ST: The reliability and validity of assessing medio-lateral patellar position: a systematic review. Man Ther, 2009 , 14 : $355-362$. [Medline] [CrossRef]

17) Shih YF, Bull AM, McGregor AH, et al.: A technique for the measurement of patellar tracking during weight-bearing activities using ultrasound. Proc Inst Mech Eng H, 2003, 217: 449-457. [Medline] [CrossRef]

18) Tennant S, Williams A, Vedi V, et al.: Patello-femoral tracking in the weight-bearing knee: a study of asymptomatic volunteers utilising dynamic magnetic resonance imaging: a preliminary report. Knee Surg Sports Traumatol Arthrosc, 2001, 9: 155-162. [Medline] [CrossRef]

19) Redmond AC, Crane YZ, Menz HB: Normative values for the Foot Posture Index. J Foot Ankle Res, 2008, 1: 6. [Medline] [CrossRef]

20) Weir JP: Quantifying test-retest reliability using the intraclass correlation coefficient and the SEM. J Strength Cond Res, 2005, 19: 231-240. [Medline]

21) Herrington L, Pearson S: The applicability of ultrasound imaging in the assessment of dynamic patella tracking: a preliminary investigation. Knee, 2008, 15: 125-127. [Medline] [CrossRef]

22) Salsich GB, Ward SR, Terk MR, et al.: In vivo assessment of patellofemoral joint contact area in individuals who are pain free. Clin Orthop Relat Res, 2003, (417): 277-284. [Medline]

23) Farrokhi S, Keyak JH, Powers CM: Individuals with patellofemoral pain exhibit greater patellofemoral joint stress: a finite element analysis study. Osteoarthritis Cartilage, 2011, 19: 287-294. [Medline] [CrossRef] 